\title{
Local Bifurcation of Steady State Solutions for A Class of Reaction-Diffusion System
}

\author{
Yupei Zhang ${ }^{1}$, Xibing $\mathrm{He}^{2}$ \\ ${ }^{1}$ Nanchang Institute of Science and Technology, Nanchang,330108, China \\ ${ }^{2}$ Department of Mathematics and Computer Science, Nanchang Normal University, \\ Nanchang,330032, China
}

Keywords:Local bifurcation; double eigenvalue;Reaction-diffusion equation; steady-state solution

Abstract.In this paper,we study local bifurcation fromthe eigenvalue $\lambda=\lambda_{9}$ with multiplicity two of the Laplacian operator for the steady-state solutions of a class of reaction-diffusion equation with Robin boundary conditions on the two-dimensional rectangular area $[0,2 \pi] \times[0, \pi]$.

\section{Introduction}

In bifurcation theory,a natural problem is whether accurate descriptions parallel to that in Crandall-Rabinowitz[1][2]theorem are still possible at eigenvalueswith multiplicity greater than one,at least in special cases[3][5].Concerning eigenvalues of higher multiplicity, [4] are known of potential operators where bifurcation takes place.Local bifurcation from the branch of trivial solutions in an equation of the form $F(\lambda, u)=\Delta u+\lambda u+f(x, u)=0$ where $\lambda \in R^{1}, \Omega \subset R^{n}$ is a bounded domain and $f(x, u)=o(u)$ as $u \rightarrow 0$ has been widely treated in the literature.

\section{Preliminaries}

In this paper,we restrict ourselves in what follows to a special case of the reaction-diffusion equation

$$
\left\{\begin{array}{lr}
\frac{\partial u}{\partial t}=\Delta u+\lambda u+f(\bar{x}, u) & \text { in } \bar{x}=(x, y) \in \Omega \\
u(0, y, t)=u(2 \pi, y, t)=0 & \forall y \in[0, \pi], t>0 \\
\frac{\partial u}{\partial n}(x, 0, t)=\frac{\partial u}{\partial n}(x, \pi, t)=0 & \forall x \in[0,2 \pi], t>0 \\
u(\bar{x}, 0)=u_{0}(\bar{x}) & t=0
\end{array}\right.
$$

where $t \in[0,+\infty), \lambda \in R^{1}$ and $f$ satisfies the two following conditions:(i) $f \in C^{3}\left(\bar{\Omega} \times R^{1}\right)$; (ii) $f(\bar{x}, 0)=f_{u}(\bar{x}, 0)=f_{\text {ии }}(\bar{x}, 0)=0, f_{\text {иии }}(\bar{x}, 0)=k \neq 0$.

It is easy to find that the question of steady state bifurcation from the double eigenvalue in(1) can be converted into the bifurcation problem of the following semi-linear elliptic equation:

$$
\left\{\begin{array}{lr}
\Delta u+\lambda u+f(\bar{x}, u)=0 & \text { in } \bar{x}=(x, y) \in \Omega \\
u(0, y)=u(2 \pi, y)=0 & \forall y \in[0, \pi](2) \\
\frac{\partial u}{\partial n}(x, 0)=\frac{\partial u}{\partial n}(x, \pi)=0 & \forall x \in[0,2 \pi]
\end{array}\right.
$$

Next, we carry on the Taylor expansion about $f$ at the point of $u=0$ and we can get that

$$
f(\bar{x}, u)=f(\bar{x}, 0)+f_{u}(\bar{x}, 0) u+\frac{1}{2 !} f_{\text {ии }}(\bar{x}, 0) u^{2}+\frac{1}{3 !} f_{\text {иии }}(\bar{x}, 0) u^{3}+o\left(u^{3}\right)=\frac{k}{6} u^{3}+o\left(u^{3}\right)=u^{3}\left(\frac{k}{6}+o(1)\right)
$$


Thus if $k>0$,we can consider the case $f(\bar{x}, u)=u^{3}(1+\theta(\bar{x}, u))$ where $\theta \in C^{1}\left(\bar{\Omega} \times R^{1}\right)$ and $\theta(\bar{x}, 0)=0$.Of course, if $k<0$, accordingly we consider $f(\bar{x}, u)=-u^{3}(1+\theta(\bar{x}, u))$.

\section{The Main Results}

Theorem 1Let $\Omega=[0,2 \pi] \times[0, \pi] \subset R^{2}$. Then there exist an $\varepsilon>0$ and a neighbourhood $U$ of $\left(\lambda_{9}, 0\right)$ in $R^{1} \times C(\bar{\Omega})$ such that the set of all steady-state bifurcation solutions of(1)in $U$ can be described as the union of four $C^{1}$ curves: $s \in(-\varepsilon, \varepsilon) \mapsto\left(\lambda_{i}(s), u_{i}(s)\right), \quad i=1, \cdots, 4$, such that

$$
\left\{\begin{array}{l}
\lambda_{i}(s)=\lambda_{9}+\sigma_{i} s^{2}+o\left(s^{2}\right) \\
u_{i}(s)=s \phi_{\alpha_{i}}+o(s)
\end{array}\right.
$$

where $\alpha_{1}=0, \alpha_{2}=\pi / 4, \alpha_{3}=\pi / 2, \alpha_{4}=3 \pi / 4$, and

$$
\begin{aligned}
& \sigma_{1}=\sigma_{3}=-9 / 16, \sigma_{2}=\sigma_{4}=-21 / 32, \text { for } k>0 \\
& \sigma_{1}=\sigma_{3}=9 / 16, \sigma_{2}=\sigma_{4}=21 / 32, \text { for } k<0 .
\end{aligned}
$$

Proof.On the basis of calculation,we label $M$ as the vector space of all eigenfunctions $A=\sin x \cos 2 y$ and $B=\sin 2 x \cos y$ associated to the double eigenvalue $\lambda_{9}=\lambda_{2,2}=\lambda_{1,4}=5$ and denote $M^{\perp}=\left\{u \in C(\bar{\Omega}): \int_{\Omega} u \phi=0, \forall \phi \in M\right\}$. After that we introduce the normalizedeigenfunction $\phi_{\alpha}(x, y)=\cos \alpha \sin x \cos 2 y+\sin \alpha \sin 2 x \cos y=\cos \alpha A+\sin \alpha B, \alpha \in[0,2 \pi)$, which is a parametric representation of all eigenfunctions $\phi$ with $\|\phi\|_{L^{2}}=\pi / \sqrt{2}$. Alongside with $\phi_{\alpha}$ weintroduce an orthogonal eigenfunction defined by $\psi_{\alpha}(x, y)=\sin \alpha \sin x \cos 2 y-\cos \alpha \sin 2 x \cos y$. Notice that $\phi_{\alpha-\pi / 2}=\psi_{\alpha}$ and $D_{\alpha} \phi_{\alpha}=-\psi_{\alpha}$. Let $\left(\lambda_{n}, u_{n}\right)$ be a sequence of solutions to (2) such that $\lambda_{n} \rightarrow \lambda_{9}$ and $u_{n} \rightarrow 0$ in $C(\bar{\Omega})$. We make the normalization: $\tilde{u}_{n}=u_{n} /\left\|u_{n}\right\|_{\infty}$. Then $\tilde{u}_{n}$ verifies the equation

$$
\left\{\begin{array}{lc}
\Delta \tilde{u}_{n}+\lambda_{n} \tilde{u}_{n} \pm \tilde{u}_{n} u_{n}^{2}(1+\theta(\bar{x}, u))=0 & \text { in } \Omega \\
\tilde{u}_{n}(0, y)=\tilde{u}_{n}(2 \pi, y)=0 & \forall y \in[0, \pi](3) \\
\frac{\partial \tilde{u}_{n}}{\partial n}(x, 0)=\frac{\partial \tilde{u}_{n}}{\partial n}(x, \pi)=0 & \forall x \in[0,2 \pi]
\end{array}\right.
$$

The first formula of (3)can be transformed into $(-\Delta)^{-1}\left[\lambda_{n} \pm u_{n}^{2}(1+\theta(\bar{x}, u))\right] \tilde{u}_{n}=\tilde{u}_{n}$, Thus that (3)is equivalent to a fixed point equation for a self-sequential compact operator in $C(\bar{\Omega})$. We all know that a compact operator can map a bounded set into a compact set,and taking into account that $\left\|\tilde{u}_{n}\right\|_{\infty}=1$,passing to a subsequence still denoted by $\tilde{u}_{n}$, we have that $\tilde{u}_{n} \rightarrow u_{0} \operatorname{in} C(\bar{\Omega})$ with $\left\|u_{0}\right\|_{\infty}=1$ and $u_{0}$ satisfies

$$
\left\{\begin{array}{lr}
\Delta u_{0}+\lambda_{9} u_{0}=0 & \text { in } \Omega \\
u_{0}(0, y)=u_{0}(2 \pi, y)=0 & \forall y \in[0, \pi] . \\
\frac{\partial u_{0}}{\partial n}(x, 0)=\frac{\partial u_{0}}{\partial n}(x, \pi)=0 & \forall x \in[0,2 \pi]
\end{array}\right.
$$

It follows that $u_{0} \in M$ and for some $\alpha \in[0,2 \pi)$ we can assume $u_{0}=c \phi_{\alpha}$ with $c=\left\|\phi_{\alpha}\right\|^{-1}$.Writing $\tilde{u}_{n}=\phi_{n}+\psi_{n}$ with $\phi_{n} \in M, \psi_{n} \in M^{\perp}$ (so that $\phi_{n} \rightarrow c \phi_{\alpha}, \psi_{n} \rightarrow 0$ ); again writing 
$t_{n}=\left\|u_{n}\right\|_{\infty}$ and simplifying the first formula of(3),we arrive that

$$
\Delta\left(\phi_{n}+\psi_{n}\right)+\lambda_{9}\left(\phi_{n}+\psi_{n}\right)+\left(\lambda_{n}-\lambda_{9}\right)\left(\phi_{n}+\psi_{n}\right) \pm t_{n}^{2}\left(\phi_{n}+\psi_{n}\right)^{3}\left(1+\theta\left(\bar{x}, t_{n}\left(\phi_{n}+\psi_{n}\right)\right)\right)=0 .
$$

Since $\phi_{n} \in M$, we get that $\Delta \phi_{n}+\lambda_{9} \phi_{n}=0$. Then the above mathematical expression can be converted to

$$
\Delta \psi_{n}+\lambda_{9} \psi_{n}+\left(\lambda_{n}-\lambda_{9}\right)\left(\phi_{n}+\psi_{n}\right) \pm t_{n}^{2}\left(\phi_{n}+\psi_{n}\right)^{3}\left(1+\theta\left(\bar{x}, t_{n}\left(\phi_{n}+\psi_{n}\right)\right)\right)=0(4)
$$

Multiplying by $\phi_{\alpha}$ and integrating by parts we obtain $\frac{\left(\lambda_{n}-\lambda_{9}\right)}{t_{n}^{2}} \int_{\Omega} \phi_{n} \phi_{\alpha} \pm$ $\int_{\Omega}\left(\phi_{n}+\psi_{n}\right)^{3}\left(1+\theta\left(\bar{x}, t_{n}\left(\phi_{n}+\psi_{n}\right)\right)\right) \phi_{\alpha}=0$ from which it follows after passing to the limit that $\lim _{n \rightarrow \infty} \frac{\lambda_{n}-\lambda_{9}}{t_{n}^{2}}=\mp c^{2} \frac{\int_{\Omega} \phi_{\alpha}{ }^{4}}{\int_{\Omega} \phi_{\alpha}{ }^{2}}$. Similarly,multiplying (4)by $\psi_{\alpha}$, integrating by parts and passing to the limit we get that $\frac{\int_{\Omega} \phi_{\alpha}{ }^{4}}{\int_{\Omega} \phi_{\alpha}{ }^{2}} \int_{\Omega} \phi_{\alpha} \psi_{\alpha}=\int_{\Omega} \phi_{\alpha}{ }^{3} \psi_{\alpha}$. We have the left hand equals 0 from $\int_{\Omega} \phi_{\alpha} \psi_{\alpha}=\int_{0}^{2 \pi} d x \int_{0}^{\pi}(\cos \alpha A+\sin \alpha B)(\sin \alpha A-\cos \alpha B) d y=0$. For the right hand,it can be checked that $\int_{\Omega} \phi_{\alpha}^{3} \psi_{\alpha}=\int_{0}^{2 \pi} d x \int_{0}^{\pi}(\cos \alpha A+\sin \alpha B)^{3}(\sin \alpha A-\cos \alpha B) d y=-\frac{3}{64} \pi^{2} \sin 2 \alpha \cos 2 \alpha$, so we obtain that the bifurcation is only possible from four values of $\alpha$,namely $\alpha=0, \pi / 4, \pi / 2,3 \pi / 4$. Notice that there are other values of $\alpha$.Since $\phi_{\pi+\alpha}=-\phi_{\alpha}$, we can find that the bifurcation occurs only at $\alpha=0, \pi / 4, \pi / 2,3 \pi / 4$. Writing $s=c t_{n}, \sigma=\mp \frac{\int_{\Omega} \phi_{\alpha}{ }^{4}}{\int_{\Omega} \phi_{\alpha}{ }^{2}}$, we have $\lim _{n \rightarrow \infty} \frac{\lambda_{n}-\lambda_{9}}{c^{2} t_{n}^{2}}=\lim _{n \rightarrow \infty} \frac{\lambda_{n}-\lambda_{9}}{s^{2}}=\sigma$, that is $\lambda_{n}=\lambda_{9}+s^{2} \sigma+o\left(s^{2}\right)$. Through calculation we can get $\sigma_{1}=\sigma_{3}=-9 / 16, \sigma_{2}=\sigma_{4}=-21 / 32$ for $k>0$. and $\sigma_{1}=\sigma_{3}=9 / 16, \sigma_{2}=\sigma_{4}=21 / 32$ for $k<0$. Besides if followsfrom(4)that $\Delta \psi_{n}+\lambda_{9} \psi_{n}=\mathrm{O}\left(t_{n}^{2}\right)$. Since $\left(\Delta+\lambda_{9}\right)^{-1}$ is a bounded linear operator from $M^{\perp}$ into itself,we can get

$$
\psi_{n}=\mathrm{O}\left(t_{n}^{2}\right), u_{n}=\left\|u_{n}\right\|_{\infty} \tilde{u}_{n}=t_{n}\left(c \phi_{\alpha+o(1)}+\mathrm{O}\left(t_{n}^{2}\right)\right)=s \phi_{\alpha+o(1)}+\mathrm{O}\left(s^{3}\right) .
$$

As a consequence of this analysis,any solution $\left(\lambda_{n}, u_{n}\right)$ near the bifurcation point $\left(\lambda_{9}, 0\right)$ has the form $\left\{\begin{array}{l}\lambda_{n}=\lambda_{9}+s^{2} \sigma+o\left(s^{2}\right) \\ u_{n}=s \phi_{\alpha+o(1)}+O\left(s^{3}\right)\end{array}\right.$. Nowwe turn to the actual construction of the bifurcated branches.Let $\alpha_{0}$ be fixed as one of the four values $\alpha=0, \pi / 4, \pi / 2,3 \pi / 4$ given above.For $s$ small we want to solve it: $\left\{\begin{array}{lc}\Delta \psi+\lambda_{9} \psi+\sigma \phi_{\alpha}+s^{2} \sigma \psi \pm\left(\phi_{\alpha}+s^{2} \psi\right)^{3}\left(1+\theta\left(\bar{x}, s \phi_{\alpha}+s^{3} \psi\right)\right)=0 & \text { in } \Omega \\ \psi(0, y)=\psi(2 \pi, y)=0 & \forall y \in[0, \pi] \text {. Denoting by } K \\ \frac{\partial \psi}{\partial n}(x, 0)=\frac{\partial \psi}{\partial n}(x, \pi)=0 & \forall x \in[0,2 \pi]\end{array}\right.$ the inverse of $\Delta$ which is a compact and linear operator from $C(\bar{\Omega})$ into itself.

For $\alpha, \sigma, \psi$ in a small neighbourhood of $\alpha_{0}, \sigma_{0}, \psi_{0}$ respectively,the above problem is equivalent to $H(\alpha, \sigma, \psi, s)=0$ where 
$H(\alpha, \sigma, \psi, s)=\psi+K\left(\lambda_{9} \psi+\sigma \phi_{\alpha}+s^{2} \sigma \psi \pm\left(\phi_{\alpha}+s^{2} \psi\right)^{3}\left(1+\theta\left(\bar{x}, s \phi_{\alpha}+s^{3} \psi\right)\right)\right)$ and $\psi_{0} \in M^{\perp}$ isthe unique solution of this equation

$$
\left\{\begin{array}{lr}
\Delta \psi+\lambda_{9} \psi+\sigma_{0} \phi_{\alpha_{0}} \pm \phi_{\alpha_{0}}{ }^{3}=0 & \text { in } \Omega \\
\psi(0, y)=\psi(2 \pi, y)=0 & \forall y \in[0, \pi] . \\
\frac{\partial \psi}{\partial n}(x, 0)=\frac{\partial \psi}{\partial n}(x, \pi)=0 & \forall x \in[0,2 \pi]
\end{array}\right.
$$

Let us apply the implicit function theorem in our setting.First we must notice that $H$ is a $C^{1}$ function ofits arguments in a neighbourhood $Q$ of $\left(\alpha_{0}, \sigma_{0}, \psi_{0}, 0\right)$ in $R^{1} \times R^{1} \times M^{\perp} \times R^{1}$. Also, $H\left(\alpha_{0}, \sigma_{0}, \psi_{0}, 0\right)=0$ and $D_{(\alpha, \sigma, \psi)} H\left(\alpha_{0}, \sigma_{0}, \psi_{0}, 0\right)(\tilde{\alpha}, \tilde{\sigma}, \tilde{\psi})=\tilde{\psi}+K\left(\lambda_{9} \tilde{\psi}+\tilde{\sigma} \phi_{\alpha_{0}}-\tilde{\alpha}\left(\sigma_{0} \psi_{\alpha_{0}} \pm 3 \phi_{\alpha_{0}}^{2} \psi_{\alpha_{0}}\right)\right)$ For some $(\tilde{\alpha}, \tilde{\sigma}, \tilde{\psi}) \in R^{1} \times R^{1} \times M^{\perp}$, we assume that $D_{(\alpha, \sigma, \psi)} H\left(\alpha_{0}, \sigma_{0}, \psi_{0}, 0\right)(\tilde{\alpha}, \tilde{\sigma}, \tilde{\psi})=0$. This means that $\tilde{\psi}$ solve the following problem

$$
\left\{\begin{array}{lc}
\Delta \tilde{\psi}+\lambda_{9} \tilde{\psi}+\tilde{\sigma} \phi_{\alpha_{0}}-\left(\sigma_{0} \psi_{\alpha_{0}} \pm 3 \phi_{\alpha_{0}}{ }^{2} \psi_{\alpha_{0}}\right) \tilde{\alpha}=0 & \text { in } \Omega \\
\tilde{\psi}(0, y)=\tilde{\psi}(2 \pi, y)=0 & \forall y \in[0, \pi] .(5) \\
\frac{\partial \tilde{\psi}}{\partial n}(x, 0)=\frac{\partial \tilde{\psi}}{\partial n}(x, \pi)=0 & \forall x \in[0,2 \pi]
\end{array}\right.
$$

Multiplying by $\phi_{\alpha_{0}}$,integrating in $\Omega$ and performing an integration by parts, we arrive at $\tilde{\sigma}=0$ since $\int_{\Omega} \phi_{\alpha}{ }^{3} \psi_{\alpha}=0$ holds.Multiplying by $\psi_{\alpha_{0}}$ instead,we get $\left(\sigma_{0} \int_{\Omega} \psi_{\alpha_{0}}{ }^{2} \pm 3 \int_{\Omega} \phi_{\alpha_{0}}^{2} \psi_{\alpha_{0}}^{2}\right) \tilde{\alpha}=0$. In view of that $\int_{\Omega} \psi_{\alpha_{0}}^{2}=\frac{\pi^{2}}{2}$ and $\int_{\Omega} \phi_{\alpha_{0}}^{2} \psi_{\alpha_{0}}^{2}=\frac{\pi^{2}}{8}-\frac{3}{64} \pi^{2} \sin ^{2} 2 \alpha_{0}$, it is easy to see that the term inside brackets is always nonzero.Thus $\tilde{\alpha}=0$ and (5)leads to $\tilde{\psi}=0$. Tosummarize, $D_{(\alpha, \sigma, \psi)} H\left(\alpha_{0}, \sigma_{0}, \psi_{0}, 0\right)(\tilde{\alpha}, \tilde{\sigma}, \tilde{\psi})$ is one-to-one and hence an isomorphism since it can be viewed as a compact perturbation of the identity. $\forall \varepsilon>0$, the implicit function theorem applies to three $C^{1}$ functions $\alpha:(-\varepsilon, \varepsilon) \rightarrow R^{1}$, $\sigma:(-\varepsilon, \varepsilon) \rightarrow R^{1}, \psi:(-\varepsilon, \varepsilon) \rightarrow M^{\perp}$ such that $\alpha(0)=\alpha_{0}, \sigma(0)=\sigma_{0}, \psi(0)=\psi_{0}$ and the set of solutions of $H(\alpha, \sigma, \psi, s)=0$ near the point $\left(\alpha_{0}, \sigma_{0}, \psi_{0}, 0\right)$ can be expressed as $(\alpha(s), \sigma(s), \psi(s), s)$ This conclusion together with the form of $\left(\lambda_{n}, u_{n}\right)$ gives in particular a unique curve of solutions to(2)such that $u(s) \sim s \phi_{\alpha_{0}}$ as $s \rightarrow 0$. Since $\alpha_{0}$ can be taken as any of the four values $0, \pi / 4, \pi / 2,3 \pi / 4$. ,we have exactly four branches of solutions near the bifurcation point $\left(\lambda_{9}, 0\right)$.The proof of the theorem is thus complete and the bifurcation graphic looks like this:
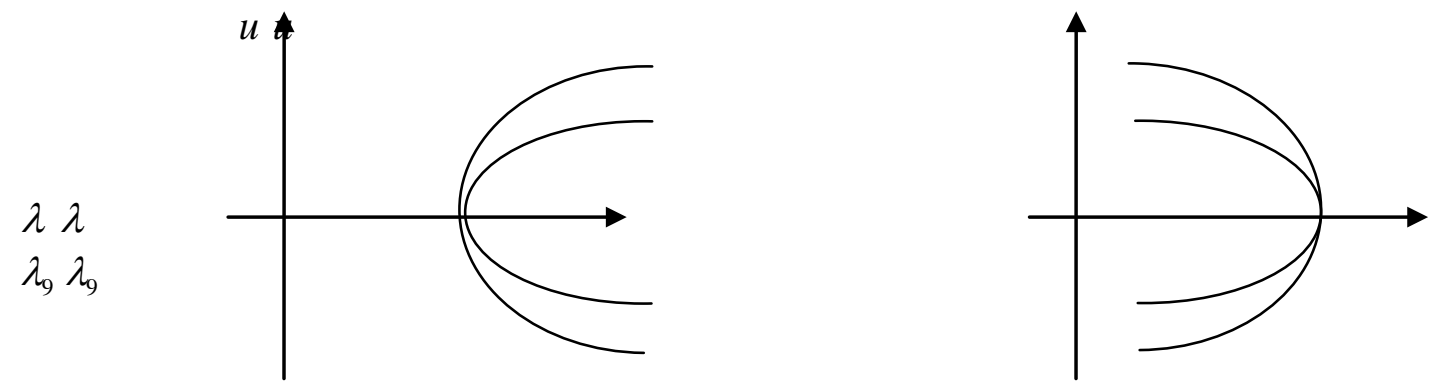

(i)for $k<0$ (ii)for $k>0$ 


\section{Conclusion}

Based on the analysis,we should mention that the nonlinearity $\pm u^{3}(1+\theta(\bar{x}, u))$ can be replaced by $\pm u^{2 m+1}(1+\theta(\bar{x}, u)), m \in N^{+}$, with no change basically in the proofs above together with mathematical induction. The next step of our work is that case $f(\bar{x}, u)= \pm u^{2}(1+\theta(\bar{x}, u))$ and the corresponding local and steady state bifurcation problem of (1).

\section{Acknowledgements}

In this paper, the research was supported by Science and Technology Research Project of Jiangxi Provincial Department of Education (No. GJJ151250), Startup Foundation for Doctors of Nanchang Normal University(No. 2015027), Nature Science Foundation of China(No. 11661057).

\section{References}

[1]Chow SN, Hale JK. Methods of bifurcation theory[M]. New York: Springer-Verlag, 1982.

[2] Crandall MG, Rabinowitz PH. Bifurcation from simple eigenvalues[J]. Journal of Functional Analysis, 1971, 8(2): 321-340.

[3]Del Pino M, García-Melián J, Musso M. Local bifurcation from the second eigenvalue of the Laplacian in a square[J]. Proceedings of the American Mathematical Society, 2003, 131(11): 3499-3505.

[4]Rabinowitz PH. A bifurcation theorem for potential operators[J]. Journal of functional Analysis, 1977, 25(4): 412-424.

[5] Shi JP. Semilinear Neumann boundary value problems on a rectangle[J]. Transactions of the American Mathematical Society, 2002, 354(8): 3117-3154. 